\title{
Determinación del Balance Catión Anión Dietario en Vacas de Preparto en Establos Lecheros de Lima y Trujillo, Perú, y su Relación con el pH Urinario
}

\author{
Determination of Anion Cation Balance in Dairy Cattle Herds of Lima And \\ Trujillo, Peru and its Relationship with Urinary PH
}

\author{
Ángel Guzmán ${ }^{2,3,4}$, Belén Montoya ${ }^{1}$, Carlos A. Gómez ${ }^{1}$
}

\section{Resumen}

\begin{abstract}
El objetivo del presente estudio fue determinar el balance catión anión dietario (DCAD) en vacas Holstein multíparas en etapa de preparto de nueve establos de Lima y Trujillo, Perú. Se analizaron muestras de concentrado y forraje para determinar la concentración de sodio, potasio, cloro y azufre, a fin de calcular el valor de DCAD mediante fórmula matemática. De manera similar, se realizó la colección de orina en 76 vacas durante la etapa de preparto para evaluar la relación del $\mathrm{pH}$ urinario con el DCAD. El valor promedio de DCAD para las dietas de los establos de Lima fue de $6.62 \pm 2.99 \mathrm{mEq} / 100 \mathrm{~g}$ MS y de $13.09 \pm 5.36 \mathrm{mEq} / 100 \mathrm{~g} \mathrm{MS}$ para los establos de Trujillo. El valor de $\mathrm{pH}$ urinario promedio fue 7.7, con un rango de 6.0 a 8.4. Se encontró una correlación positiva de 0.331 entre los valores de DCAD y $\mathrm{pH}$ urinario.
\end{abstract}

Palabras clave: $\mathrm{DCAD}$, preparto, $\mathrm{pH}$, vacas

\section{Abstract}

The aim of the present study was to determine the Dietary Cation-Anion Difference (DCAD) concentration in pre-partum diets from nine dairy farms of Lima and Trujillo, Peru. Feed and forage samples were analysed for sodium, potassium, chloride and sulphur in order to calculate DCAD values by mathematical formula. Similarly, urine samples were

${ }^{1}$ Facultad de Zootecnia, Universidad Nacional Agraria La Molina, Lima, Perú

${ }^{2}$ Unidad de Investigación y Desarrollo, Battilana Nutrición SAC, Lima, Perú

${ }^{3}$ E-mail: atecnica@battilana.biz

${ }^{4}$ Proyecto financiado por el Fondo para la Innovación, Ciencia y Tecnología (FINCyT)

Recibido: 1 de setiembre de 2015

Aceptado para publicación: 10 de junio de 2016 
collected from 76 cows during the pre-partum period to evaluate the relationship between urinary $\mathrm{pH}$ and DCAD. The mean DCAD value was $6.62 \pm 2.99 \mathrm{mEq} / 100 \mathrm{~g}$ DM for diets of dairy farms in Lima and $13.09 \pm 5.36 \mathrm{mEq} / 100 \mathrm{~g}$ DM for diets of dairy farms in Trujillo. Urine $\mathrm{pH}$ mean was 7.7, ranging from 6.0 to 8.48. The correlation between DCAD and urine $\mathrm{pH}$ values was 0.331 .

Key words: $\mathrm{DCAD}$, prepartum, $\mathrm{pH}$, cows

\section{INTRODUCCIÓN}

El balance catión-anión dietario (DCAD) es un concepto relativamente nuevo en la nutrición y alimentación de ganado lechero de alta producción en el Perú. Según Lean et al. (2006), se define como la suma de los cationes sodio $\left(\mathrm{Na}^{+}\right)$y potasio $\left(\mathrm{K}^{+}\right)$ menos la sumatoria de los aniones cloro $\left(\mathrm{Cl}^{-}\right)$ y azufre $\left(S^{-}\right)$en miliequivalentes sobre 100 gramos de materia seca del alimento $(\mathrm{mEq} /$ $100 \mathrm{~g} \mathrm{MS})$.

El concepto DCAD se basa en el principio de la electroneutralidad y postula que un ingreso neto de cualquier catión o anión dentro del animal resulta en una perturbación del estado ácido-base (Stewart, citado por Espino et al., 2005). En el caso de vacas lecheras, estas pueden ingresar en un estado metabólico de acidosis cuando la cantidad de aniones absorbibles predominan o pasan a un estado metabólico alcalino en el momento que existe una mayor cantidad de cationes absorbibles (Moore et al., 2000).

Diversos estudios demuestran que alterando el DCAD del alimento ofrecido durante el periodo preparto se puede favorecer un estado metabólico de ligera acidosis capaz de prevenir la hipocalcemia (Tucker et al., 1992; Goff y Horst, 1997). Asimismo, Horst et al. (1997) reportan que para reducir el tiempo y severidad de hipocalcemia se requiere de un DCAD entre -100 y $-50 \mathrm{mEq} /$ $\mathrm{kg}$ MS, mientras que otros autores sugieren valores variables de DCAD que pueden ser negativos o cercanos a cero (Sharif et al.,
2010). Por otro lado, Gaynor et al. (1989) reportan una correlación positiva de DCAD con $\mathrm{pH}$ urinario, sugiriendo el uso de $\mathrm{pH}$ en orina como herramienta de monitoreo para la identificación de animales que puedan estar sufriendo estos trastornos metabólicos. En ese sentido, Goff y Horst (1997) sugieren valores de $\mathrm{pH}$ en orina de 5.5 a 6.2 en vacas Jersey y de 6.2 a 6.8 en vacas Holstein, con el fin de reducir incidencias de hipocalcemia, y valores de $\mathrm{pH}$ mayores de 5.5 para no causar una acidosis metabólica clínica.

Si bien el concepto de DACD y su uso en establos lecheros de alta producción han sido documentados a nivel mundial, en el Perú no existen evaluaciones que indiquen el estatus actual de DCAD dietario ni su posible relación con el $\mathrm{pH}$ urinario. Por tal motivo, el presente estudio busca determinar el estatus de DCAD en dietas de vacas en etapa de preparto de establos de las cuencas de Lima y Trujillo, así como establecer la relación de DCAD con $\mathrm{pH}$ urinario.

\section{Materiales y Métodos}

\section{Establos y Criterios de Selección}

El presente estudio se llevó a cabo en dos cuencas lecheras de la costa peruana: la cuenca de Lima, que presenta una temperatura promedio de $24^{\circ} \mathrm{C}$, y la cuenca de Trujillo, con temperatura promedio anual de $18{ }^{\circ} \mathrm{C}$.

La investigación se realizó entre octubre de 2013 y mayo de 2014 e incluyó dos evaluaciones: la determinación del estatus de 
DCAD en dietas de vacas en etapa de preparto, y el establecimiento de la posible relación existente de DCAD y el pH urinario.

Los ensayos se realizaron en seis establos de Lima y tres de Trujillo. Los nueve establos trabajan bajo un sistema de crianza estabulada intensiva y cuentan en promedio con una población de 584 vacas de raza Holstein con edades entre 3 y 7 años. De estas, entre el 10 y $15 \%$ se encuentran en etapa de preparto. La alimentación se basa en forraje y concentrado; sin embargo, el tipo de forraje y concentrado es variable dependiendo de la disponibilidad de insumos, el manejo de cada establo y la cuenca. Así, la base forrajera en Lima se basa en chala de maíz, ensilado y panca, en tanto que en Trujillo se compone de chala de maíz, ensilado y broza de espárrago. Asimismo, la cantidad de alimento suministrado en cada establo es variable.

Se evaluó el $15 \%$ de las vacas multíparas en preparto por establo, disponiéndose de un total de 79 vacas para el estudio. El procedimiento para la selección de vacas fue probabilístico y de selección al azar. La producción de leche en los nueve establos era muy variable, dependiendo del número de lactación, estación del año, sistema de alimentación, número de ordeños, etc. Para este estudio se consideraron los animales que no estaban sometidos a ordeño por encontrarse en el periodo de preparto (periodo seco).

\section{Determinación de DCAD Dietario}

Se recolectaron muestras del concentrado de preparto y forraje en cada establo, obteniéndose 12 muestras de la cuenca de Lima y seis de la cuenca de Trujillo. Para los establos de Lima, la proteína cruda (PC) con base a materia seca varió entre 13 y $29 \%$ en tanto que en el forraje presentó valores de 7 a 9\%. Para los establos de Trujillo, la PC del concentrado varió de 17 a $21 \%$, en tanto que en el forraje fue de 5 a $8 \%$.
Las muestras fueron llevadas al laboratorio para el análisis de minerales. Se empleó la Metodología Volumétrica II (AOAC $\left.935.05,17^{\text {th }} \mathrm{ed}, 2000\right)$ para la determinación de cloruros, en tanto que para el análisis de sodio, potasio y azufre se empleó la metodología de emisión espectrométrica para análisis de elementos y residuos en agua (EPA, 1994). Los resultados fueron obtenidos en gramos de macroelemento por 100 gramos de materia seca.

Para la determinación de DACD se empleó la ecuación presentada por Oetzel (1991), donde DCAD $(\mathrm{mEq} / 100 \mathrm{~g} \mathrm{MS})=$ $\% \mathrm{Na} / 0.023+\% \mathrm{~K} / 0.039-\% \mathrm{Cl} / 0.035-\% \mathrm{~S} /$ 0.016 .

Los valores de sodio, potasio cloro y azufre de la ración total de cada establo se introdujeron en la ecuación, obteniéndose el DCAD dietario de la ración de preparto de cada establo expresado en mEq/100 g MS.

Las proporciones de concentrado y base forrajera en MS que componen una ración total fueron bastante variables y dependientes tanto de la cuenca como del establo. Las proporciones fueron de 35 a $40 \%$ para concentrados y de 60 a $65 \%$ para forrajes. Para efectos del presente estudio se consideró que la ración total estuvo compuesta de $40 \%$ de concentrado y $60 \%$ de base forrajera en MS. Esto permite una estimación aproximada del aporte en minerales de cada componente de la ración. Por lo tanto, el valor de DCAD de la ración total es la sumatoria que expresa esos porcentajes de cada componente mineral de la ración total.

\section{Evaluación de pH Urinario}

Las muestras de orina se obtuvieron mediante estimulación manual de la vulva, recolectando la orina en vasos esterilizados de $100 \mathrm{ml}$. El muestreo fue semanal durante las tres semanas previas al parto. Las muestras fueron evaluadas in situ de manera individual, utilizando un potenciómetro digital 
Cuadro 1. Valores porcentuales promedio de sodio, potasio, cloro y azufre, y DCAD ${ }^{1}$ $(\mathrm{mEq} / 100 \mathrm{~g}$ MS) en concentrado, forraje y ración de preparto suministrados a vacas lecheras en nueve establos lecheros de Lima y Trujillo

\begin{tabular}{lccccc}
\hline & $\begin{array}{c}\mathrm{Na} \\
(\%)\end{array}$ & $\begin{array}{c}\mathrm{K} \\
(\%)\end{array}$ & $\begin{array}{c}\mathrm{Cl} \\
(\%)\end{array}$ & $\begin{array}{c}\mathrm{S} \\
(\%)\end{array}$ & $\begin{array}{c}\text { DCAD } \\
(\mathrm{mEq} / 100 \mathrm{~g} \text { MS })\end{array}$ \\
\hline Lima & & & & & \\
Concentrado & $0.22 \pm 0.23$ & $1.38 \pm 0.21$ & $0.49 \pm 0.35$ & $0.36 \pm 0.06$ & $8.09 \pm 1.95$ \\
$\begin{array}{l}\text { Forraje } \\
\text { Ración }\end{array}$ & $0.11 \pm 0.09$ & $1.22 \pm 0.85$ & $0.60 \pm 0.58$ & $0.21 \pm 0.09$ & $5.64 \pm 3.67$ \\
Trujillo & & & & & $6.62 \pm 2.99$ \\
Concentrado & $0.12 \pm 0.01$ & $1.52 \pm 0.26$ & $0.24 \pm 0.04$ & $0.45 \pm 0.11$ & $9.32 \pm 0.96$ \\
$\begin{array}{l}\text { Forraje } \\
\text { Ración }\end{array}$ & $0.04 \pm 0.02$ & $1.23 \pm 0.43$ & $0.36 \pm 0.04$ & $0.12 \pm 0.02$ & $15.61 \pm 9.57$ \\
\hline${ }^{1}$ Balance catión-anión dietario & & & & & $13.09 \pm 5.36$ \\
\hline
\end{tabular}

Hanna, modelo HI98128, calibrado al inicio de cada medición con soluciones buffer de 4.01 y 7.01 (calibración de dos puntos).

\section{Análisis Estadístico}

Para la determinación del estatus del DCAD de las dietas de preparto, se realizó un análisis descriptivo (promedio, desviación estándar) de los valores de DCAD de forraje, concentrado y dieta de cada establo. Para establecer la posible relación entre DCAD y el pH urinario, se realizó un análisis de correlación, así como un análisis de regresión lineal, empleando el programa estadístico Minitab 15 para Windows.

\section{Resultados y Discusión}

\section{Status de DCAD en dietas de vacas en preparto}

Los valores promedios de DCAD de las dietas de preparto, forraje y concentrado suministrado en establos de las cuencas de Lima y Trujillo se presentan en el Cuadro 1. Los valores de DCAD de las dietas de Lima y Trujillo fueron de $+6.62 \mathrm{y}+13.09$, respectivamente; resultados similares a los reportados por Leclerc y Block (1989) de +22 a +6.2 para dietas de preparto, y por Tucker et al. (1992) de +5.99 a +10.19 . No obstante, los valores difieren a los indicados por Beede (2005), que recomienda un DCAD negativo o cercano a 0 , y de los valores de Horst et al. (1997), quienes reportan valores de DCAD entre -5.0 y $-10.0 \mathrm{mEq} / 100 \mathrm{~g}$ MS como niveles óptimos.

Valores de DCAD catiónicos no son recomendados en la alimentación de vacas en preparto, pues en esta etapa se desea provocar una acidificación digestiva y metabólica que permita las condiciones óptimas para la circulación adecuada de calcio en el organismo (Goff et al., 1991).

Valores de DCAD positivos en las dietas pueden deberse al uso de insumos con elevados niveles de cationes en la alimentación de ganado vacuno lechero bajo crianza intensiva (Sánchez, 1999). Esto coincide con la situación actual de los establos de Lima y 
Trujillo, donde las dietas de los establos de Lima y Trujillo se basan principalmente en el uso de maíz chala, maíz amarillo, afrecho y torta de soya que presentan valores de DCAD $\mathrm{de}+16.5,+3.1,+18 \mathrm{y}+35 \mathrm{mEq} / 100 \mathrm{~g} \mathrm{MS}$, respectivamente (NRC, 2001). Una estrategia para obtener niveles de DCAD negativos o cercanos a cero en la dieta puede ser el suministro de dietas aniónicas que permitan un mayor ingreso de cloruros y sulfatos, logrando así una acidificación del medio interno que favorezca la excreción de calcio por la orina (Oetzel et al., 1991). Al reducirse los niveles de calcio en sangre, se genera la liberación de la hormona paratiroidea y 1.25 dihidroxivitamina $\mathrm{D}$, que estimulan la liberación de calcio óseo para aumentar finalmente la concentración de calcio en sangre (Goff et al., 1991).

Se observan valores promedio de DCAD dietario más cercano a cero en establos de la cuenca de Lima con respecto a establos de la cuenca de Trujillo, aunque con una mayor variabilidad entre ellos, debido a que los establos de Lima son más heterogéneos al momento de formular sus dietas.

\section{Relación de DCAD y pH Urinario}

El valor de $\mathrm{pH}$ urinario promedio fue de 7.7 y con rango de 6.0 a 8.48. Roche et al. (2005) reportan valores similares de $\mathrm{pH}$ urinario para raciones con DCAD entre -12 y $+69 \mathrm{mEq} / 100 \mathrm{~g} \mathrm{MS}$. Esta variabilidad se puede explicar por los cambios en la concentración de calcio en la orina a medida que se acerca el día del parto. A pesar de que las vacas pueden estar recibiendo dietas con DCAD negativo, una hipocalciuria producto de la resorción del pool de calcio, ante su demanda para la producción de leche, puede provocar un incremento del $\mathrm{pH}$ urinario (Joyce et al., 1997). La alta variabilidad de $\mathrm{pH}$ urinario puede explicarse, además, por el momento en que se realiza la colección de orina (Goff y Horst, 1997), recomendándose hacerlo entre 2 y 6 horas después de la alimentación. Pese a estos inconvenientes, la medición de
$\mathrm{pH}$ urinario puede ser usada como herramienta práctica para monitorear la acidificación que se produce en sangre por una suplementación con aniones (Goff et al., 2004).

En lo que se refiere a la relación de DCAD dietario y $\mathrm{pH}$ urinario, se aprecia una correlación de +0.331 entre ambas variables. Este resultado concuerda con el estudio de Roche et al. (2005), quienes aseguran que el valor de $\mathrm{pH}$ en sangre y orina se incrementa conforme aumenta el valor de DCAD dietario. Hu et al. (2007) indican valores de correlación por encima de +0.860 , lo mismo que Charbonneau et al. (2006), quienes al realizar un meta análisis de 22 trabajos, encuentran una correlación de +0.85 para la relación DCAD con $\mathrm{pH}$ urinario. Estas diferencias en los niveles de correlación se debe a la alta variabilidad observada, tanto en los valores de DCAD dietario como en los valores de $\mathrm{pH}$ urinario del presente estudio. Hay que tener en consideración que, además de la dieta, factores como la presencia de gérmenes en la orina, alcalosis respiratoria, alcalosis metabólica, acidosis metabólica, medicación acidificante (cloruro de amonio), diarreas graves, insuficiencia respiratoria y enfisema podrían afectar el $\mathrm{pH}$ urinario.

La Figura 1 muestra la regresión lineal existente entre DCAD dietario y $\mathrm{pH}$ urinario. El coeficiente de determinación de 0.11 indica que la ecuación obtenida solo explica el $11 \%$ del valor de la variable. Si bien existe una limitada relación entre DCAD y $\mathrm{pH}$ urinario en el presente estudio, la literatura indica que el $\mathrm{pH}$ en orina es una herramienta de monitoreo para programas de suplementación con sales aniónicas (Gaynor et al., 1989).

\section{Conclusiones}

- El balance catión-anión dietario (mEq/ $100 \mathrm{~g}$ MS) promedio de las nueve raciones de preparto utilizadas en establos le- 


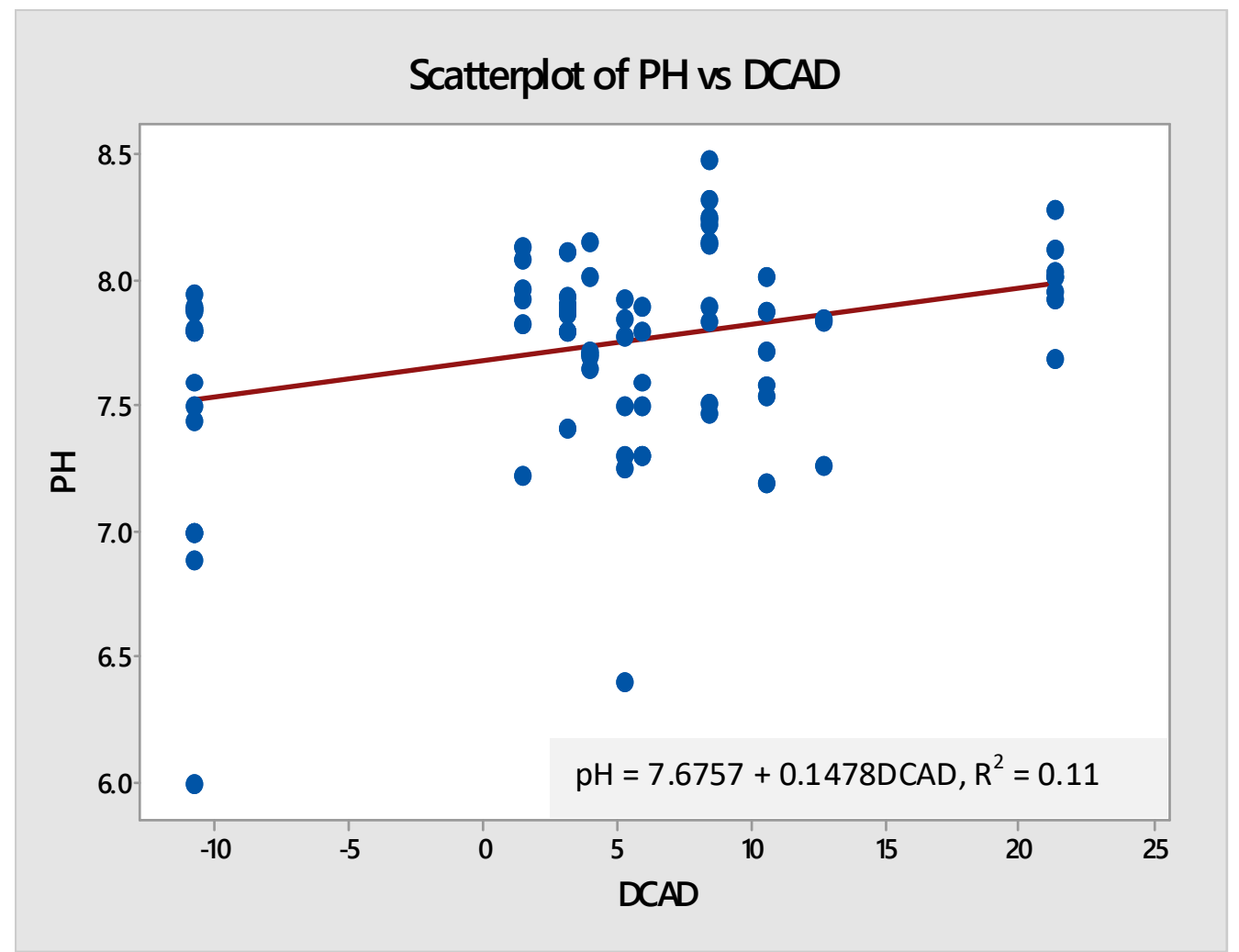

Figura 1. Regresión lineal entre DCAD dietario y $\mathrm{pH}$ urinario de vacas Holstein en la etapa de preparto $(\mathrm{n}=76)$ de establos de Lima y Trujillo

cheros de Lima y Trujillo fue de $9.1 \pm$ 6.1 en un rango entre 1.5 y 21.3 .

- La correlación entre el balance catiónanión dietario (DCAD) y nivel de $\mathrm{pH}$ urinario fue de +0.331 , indicando una limitada relación entre ambas variables.

\section{Agradecimientos}

Los autores agradecen al Dr. Eduardo Fuentes por la colaboración prestada en la revisión del presente artículo.

\section{Literatura Citada}

1. AOAC-935.05. 2013. AOAC Official Method 935.05 Chloride in plants volumetric method II. [Internet].
Available in: http://files.foodmate.com/ 2013/files 2910.html

2. Beede DK. 2005. Formulation of rations with optimal cations and anions for lactation. In: Proc Tri-State Dairy Nutrition Conference. Fort Wayne, Indiana, USA.

3. Charbonneau E, Pellerin D, Oetzel GR. 2006. Impact of lowering dietary cation-anion difference in nonlactating dairy cows: a meta-analysis. J Dairy Sci 89: 537-548. doi: 10.3168/jds.S00220302(06)72116-6

4. [EPA] United States Environmental protection Agency. 1994. Method 200.7, Revision 4.4: determination of metals and trace elements in water and wastes by inductively coupled plasmaatomic emission spectrometry. [Internet]. 
Disponible en: https://www.epa.gov/ sites/production/files/2015-08/ documents/method_200-7_rev_44_1994.pdf

5. Espino L, Suárez ML, Santamarina G, Goicoa A, Fidalgo LE. 2005. Utilización de las sales aniónicas en la prevención de la paresia puerperal hipocalcémica. Arch Med Vet 37: 7-13. doi: 10.4067/S0301-732X2005000100002

6. Gaynor PJ, Mueller FJ, Miller JK, Ramsey N, Goff JP, Horst RL. 1989. Parturient hypocalcemia in Jersey cows fed alfalfa haylage-based diets with different cation to anion ratios. J Dairy Sci 72: 2525-2531. doi: 10.3168/ jds.S0022-0302(89)79392-9

7. Goff JP, Horst RL, Mueller FJ, Miller JK, Kiess GA, Dowlen HH. 1991. Addition of chloride to a prepartal diet high in cations increases 1,25dihydroxyvitamin $\mathrm{D}$ response to hypocalcemia preventing milk fever. J Dairy Sci 74: 3863-3871. doi: 10.3168/ jds.S0022-0302(91)78579-2

8. Goff JP, Horst RL. 1997. Effects of the addition of potassium or sodium, but not calcium, to prepartum rations on milk fever in dairy cows. J Dairy Sci 80: 176186. doi: $10.3168 /$ jds.S00220302(97)75925-3

9. Goff JP, Ruiz R, Horst RL. 2004. Relative acidifying activity of anionic salts commonly used to prevent milk fever. J Dairy Sci 87: 1245-1255. doi: 10.3168/ jds.S0022-0302(04)73275-0

10. Horst RL, Goff JP, Reinhardt TA, Buxton DR. 1997. Strategies for preventing milk fever in dairy cattle. J Dairy Sci 80: 1269-1280. doi: 10.3168/ jds.S0022-0302(97)76056-9

11. Hu W, Kung L, Murphy MR. 2007. Relationships between dry matter intake and acid-base status of lactating dairy cows as manipulated by dietary cationanion difference. Anim Feed Sci Tech 136: 216-225. doi: 10.1016/ j.anifeedsci.2006.09.013
12. Joyce PW, Sanchez WK, Goff JP. 1997. Effect of anionic salts in prepartum diets based on alfalfa. J Dairy Sci 80: 2866-2875. doi: 10.3168/ jds.S0022-0302(97)76251-9

13. Lean IJ, DeGaris PJ, McNeil DM, Block E. 2006. Hypocalcemia in dairy cows: meta-analysis and dietary cation anion difference theory revisited. J Dairy Sci 89: 669-684. doi: 10.3168/jds.S00220302(06)72130-0

14. Leclerc H, Block E. 1989. Effects of reducing dietary cation-anion balance for prepartum dairy cows with specific reference to hypocalcemic parturient paresis. Can J Anim Sci 69: 411-423. doi: 10.4141/cjas89-046

15. Moore SJ, VandeHaar MJ, Sharma BK, Pilbeam TE, Beede DK, Bucholtz HF, Liesman JS, et al. 2000. Effects of altering dietary cation-anion difference on calcium and energy metabolism in peripartum cows. J Dairy Sci 83: 2095-2104.

16. [NRC] National Research Council. 2001. Nutrient requirements of dairy cattle. $7^{\text {th }}$ Rev Ed. Washington, USA: NRC. $381 \mathrm{p}$.

17. Oetzel GR, Fettman MJ, Hamar DW, Olson JD. 1991. Screening of anionic salts for palatability, effects on acid-base status, and urinary calcium excretion in dairy cows. J Dairy Sci 74: 965-971. doi: 10.3168/jds.S0022-0302(91)78245-3

18. Oetzel GR. 1991. Meta-analysis of nutritional risk factors for milk fever in dairy cattle. J Dairy Sci 74: 3900-3912. doi: 10.3168/jds.S0022-0302(91)78583-4

19. Roche JR, Petch S, Kay JK. 2005. Manipulating the dietary cation-anion difference via drenching to earlylactation dairy cows grazing pasture. J Dairy Sci 88: 264-276. doi: 10.3168/ jds.S0022-0302(05)72684-9

20. Sanchez WK. 1999. Another new look at DCAD for the prepartum dairy cow. In: Proc Mid-South Ruminant Nutrition Conference. Dallas-Fort Worth, Texas, USA. 
21. Sharif M, Shahzad MA, Mahr-unNisa, Sarwar M. 2010. Dietary cation anion difference: impact on productive and reproductive performance in animal agriculture. Afr J Biotechnol 9: 79767988.
22. Tucker WB, Hogue JF, Adams GD, Aslam M, Shin IS, Morgan G. 1992. Influence of dietary cation-anion balance during the dry period on the occurrence of parturient paresis in cows fed excess calcium. J Anim Sci 70: 1238-1250. 